\title{
Collective Excitations of a "Gravitationally" Self-Bound Bose Gas
}

\author{
S. Giovanazzi ${ }^{(a)}$, G. Kurizki ${ }^{(a)}$, I. E. Mazets ${ }^{(b)}$, and S. Stringari ${ }^{(c)}$ \\ (a) Department of Chemical Physics, Weizmann Institute of Science, 76100 Rehovot, Israel, \\ (b) Ioffe Physico-Tecnical Institute, 194021 St.Petersburg, Russia, \\ (c) Dipartimento di Fisica, Universita di Trento and Istituto Nazionale per la Fisica della Materia, I-38050 Povo, Italy.
}

(November 9, 2018)

We investigate the collective excitations of an atomic BoseEinstein condensate in the self-binding regime produced by electromagnetically induced "gravity" (1/r attraction). Analytical expressions for the frequencies of the monopole and quadrupole modes are obtained at zero temperature, using the sum-rule approach, and compared with the exact results available in the Thomas-Fermi limit. The low-energy dynamics of such condensates is shown to be dominated by the effective "plasma" frequency. An analog of the Jeans gravitational instability is analyzed.

PACS number(s): 03.75.Fi, 34.20.Cf, 34.80.Qb, 04.40.-b

Introduction - Recently, a new kind of atomic BoseEinstein condensates has been proposed [1]. It has been shown that particular configurations of intense, offresonant electromagnetic fields mimic the gravitational attraction between atoms located well within the wavelength of these fields, giving rise to scenarios very different from the ones characterizing usual Bose-Einstein condensates 22.37. The interatomic potential is of the form $-u / r$, where the gravitational coupling $u=G m^{2}$ is replaced by

$$
u=\frac{11}{60 \pi \epsilon_{0}^{2} c} \alpha^{2} q^{2} I
$$

Here the total laser intensity and wavenumber are denoted by $I$ and $q$, respectively, and $\alpha$ is the atomic polarizability at the frequency $c q$ [1]. For a sufficiently strong induced "gravity" the BEC becomes self-bound, i.e. stable in the absence of an external confining potential [ $[$ ]. Such a self-bound system may become a laboratory ana$\log$ of a non-relativistic Bose star [5], a self-gravitating system that balances gravity with the "quantum pressure" corresponding to the zero-point kinetic energy fluctuation. In the self-bound condensate considered in Ref. [1] the induced "gravity" can be also balanced by the short-range interatomic repulsion.

It is known that the long-wavelength collective excitations of a spatially-uniform system of both fermions (e.g., the electron gas) and bosons [6], interacting via a repulsive potential of the form $u / r$, are characterized by a spectral gap, fixed by the "plasma" frequency

$$
\omega_{p}^{2}=4 \pi u n / m
$$

where $n$ is the particle density. In this paper we show that also in the case of a self-bound atomic BEC, interacting via an attractive $-u / r$ potential, the collective oscillations are characterized by an effective "plasma" frequency $\omega_{p}$ of the form (2), with a spatially-averaged density $n$ and the positive coupling constant $u$ [Eq. (1i)]. Its correspondence with the fundamental oscillation frequency of white dwarfs and neutron stars [7] is pointed out and the analog of the Jeans gravitational instability is explicitly analyzed.

Generalized Gross-Pitaevskii equation - The GrossPitaevskii equation [8] for the order parameter $\Psi(\mathbf{r}, t)$ can be obtained starting from

$$
i \hbar \frac{\partial \Psi}{\partial t}=\frac{\delta}{\delta \Psi^{*}} H_{\mathrm{tot}} .
$$

Here the total mean-field energy functional $H_{\text {tot }}=H_{\text {kin }}+$ $H_{\text {ho }}+H_{\text {grav }}+H_{\mathrm{s}}$ consists of: (a) the kinetic energy, $H_{\text {kin }}=\int d \mathbf{r}\left(\hbar^{2} / 2 m\right)|\nabla \Psi|^{2}$; (b) the harmonic-trap energy, $H_{\text {ho }}=\int V_{\text {ho }} n d \mathbf{r}$, where $n=|\Psi(\mathbf{r}, t)|^{2}$ is the atomic density and $V_{\mathrm{ho}}=m \omega_{0}^{2} r^{2} / 2$ is an isotropic harmonic external potential; (c) the mean-field energy due to the electromagnetically induced "gravity", $H_{\text {grav }}=$ $(1 / 2) \int n \Phi d \mathbf{r}$, where $\Phi$ is the "gravitational potential" solution of the Poisson equation

$$
\nabla^{2} \Phi=4 \pi u n,
$$

with the boundary condition $\Phi=0$ for $|r| \rightarrow \infty$; (d) the mean-field contribution due to the short-range scattering, $H_{\mathrm{s}}=(g / 2) \int n^{2} d \mathbf{r}$, where $g=4 \pi a \hbar^{2} / m$, a being the swave scattering length.

By expressing the complex BEC order parameter through the condensate density $n$ and phase $\phi$ as $\Psi=$ $\sqrt{n} \exp (i \phi)$, we can write Eq. (3) as a coupled set of collisionless hydrodynamic equations:

$$
\frac{\partial n}{\partial t}+\nabla(n \mathbf{v})=0
$$

$m \frac{\partial \mathbf{v}}{\partial t}+\nabla\left(\frac{m v^{2}}{2}-\frac{\hbar^{2} \nabla^{2} \sqrt{n}}{2 m \sqrt{n}}+g n+V_{\text {ho }}+\Phi\right)=0$.

The last equation establishes the irrotational property of the superfluid velocity field defined by $\mathbf{v}=\hbar \nabla \phi / m$. Similar equations have been derived in the case of harmonically trapped Bose-Einstein condensates interacting via zero-range forces [9].

Jeans-like instability - In the absence of external potentials, Eqs. (5) and (6) admit a stationary solution with constant density $n$. Such a solution is, however, dynamically unstable, as can be seen directly from the 
Bogoliubov dispersion relation [10] corresponding to (5) and (6):

$$
\omega_{k}^{2}=\frac{\hbar^{2} k^{4}}{4 m^{2}}+c_{s}^{2} k^{2}-\omega_{p}^{2} .
$$

In (7) $c_{s}=\sqrt{g n / m}$ is the Bogoliubov speed of sound and the plasma frequency $\omega_{p}$ is given by (2). The occurrence of imaginary frequencies $\omega_{k}$ for small values of $k$ reveals the existence of an instability, which resembles the Jeans gravitational instability [5, 11]. Assuming $\omega_{p} \ll m c_{s}^{2}$, the minimal wavelength ensuring a real value for the frequency (7) is given by

$$
\lambda_{J}=2 \pi c_{s} / \omega_{p}=2 \pi\left(\hbar^{2} a / m u\right)^{1 / 2} .
$$

In a "self-gravitating" BEC, both $\omega_{p}$ and $c_{s}$ scale as $n^{1 / 2}$, so that the critical wavelength $\lambda_{J}$ is independent of the density, as explicitly revealed by (8).

Besides these unstable solutions, Eqs. (5) and (6) admit a stable self-bound solution for any finite number $\mathrm{N}$ of atoms. In this case long-wavelength fluctuations, which can trigger the Jeans instability, are excluded by the finite size of the system. Remarkably, the ground-state density of a self-bound Bose cloud, in the limit in which the kinetic energy can be neglected (Thomas-Fermi limit for Bose gases), has the analytic profile [1]

$$
n_{0}(\mathbf{r})=\frac{N}{4 R_{0}^{2}} \frac{\sin \left(\pi r / R_{0}\right)}{r} \theta\left(R_{0}-r\right)=0,
$$

where the radius $R_{0}=\lambda_{J} / 2$ is fixed by the Jeans wavelength and $\theta$ is the Heaviside function.

Sum rule approach - The quadrupole and monopole excitation frequencies at zero temperature can be estimated using a sum-rule approach 9, 12]. The calculation of the oscillation frequencies $\omega$ is based on the ratio

$$
\hbar^{2} \omega^{2}=m_{3} / m_{1}
$$

between the third moment (cubic-energy weighted) and the first moment (energy-weighted) of the dynamic structure factor: $m_{p}=\sum_{n}\left(\hbar \omega_{n 0}\right)^{p}|<0| F|n>|^{2}(p=1,3)$. Here $\hbar \omega_{n 0}$ is the excitation energy of the state $|n\rangle$ and $F$ is an excitation operator chosen similarly to [9]: $F=\sum_{i=1}^{N} r_{i}^{2}$ or $F=\sum_{i=1}^{N} r_{i}^{2} Y_{2 m}$, for the monopole or quadrupole operators, respectively. The moments $m_{1}$ and $m_{3}$ can be reduced to the form of commutators involving the Hamiltonian of the system and evaluated in terms of ground-state expectation values. One then obtains the following results:

$$
\begin{aligned}
\omega_{M}^{2} & =\frac{4 H_{\text {kin }}+4 H_{\mathrm{ho}}+H_{\text {grav }}+9 H_{\mathrm{s}}}{N m\left\langle r^{2}\right\rangle} \\
& =\frac{2 H_{\text {kin }}+6 H_{\mathrm{ho}}+6 H_{\mathrm{s}}}{N m\left\langle r^{2}\right\rangle}, \\
\omega_{Q}^{2} & =\frac{4 H_{\mathrm{kin}}+4 H_{\mathrm{ho}}-4 / 5 H_{\text {grav }}}{N m\left\langle r^{2}\right\rangle},
\end{aligned}
$$

where, in deriving the second equation (11), we have used the virial identity

$$
2 H_{\text {kin }}-2 H_{\text {ho }}+H_{\text {grav }}+3 H_{\mathrm{s}}=0 .
$$

The frequencies $\omega_{M}$ and $\omega_{Q}$, given by (11) and (12), are always real for positive values of the scattering length. If the expectation values of Eqs. (11) and (12) are evaluated using the exact ground state, they provide a rigorous upper bound to the lowest excitation frequencies for a given multipole. This approach has been shown to provide an excellent description of the crossover between the non-interacting and the Thomas-Fermi regimes in the case of condensates interacting via zero-range forces [3].

A useful analytical estimate for the expectation values of Eqs. (11) and (12) can be obtained by replacing the exact ground state with the gaussian wavefunction $\left.\Psi=N^{\frac{1}{2}} \exp \left(-r^{2} / 2 w^{2}\right)\right) / \pi^{\frac{3}{4}} w^{\frac{3}{2}}$. Here $w$ is a variational parameter that can be calculated by minimizing the total energy $H_{\text {tot }}$ and is related to the expectation value $\left\langle r^{2}\right\rangle=(3 / 2) w^{2}$. The various contributions to the energy $H_{\text {tot }}$ then take the form: $H_{\text {kin }}=3 N \hbar^{2} / 4 m w^{2}, H_{\text {ho }}$ $=N m \omega_{0}^{2} w^{2} / 4, H_{\mathrm{s}}=a \hbar^{2} N^{2} / \sqrt{2 \pi} m w^{3}$ and $H_{\text {grav }}=$ $-(1 / \sqrt{2 \pi}) u N^{2} / w$. The gaussian ansatz allows us to describe analytically the various regimes in the "phase diagram" of the problem [1]. It is noteworthy that the monopole and quadrupole frequencies obtained by the gaussian ansatz coincide with the ones derivable from a time-dependent variational calculation in a gaussian basis [13].

Low-energy excitations in a self-bound BEC - In the following we calculate the monopole and quadrupole excitation frequencies for a self-bound BEC from Eqs. (11) and (12), using, for the ground state, either the exact solution of the Gross-Pitaevskii equation or the gaussian approximation. In these calculations we are interested in the effects of s-wave scattering, measured by the dimensionless parameter

$$
s=m u N^{2} a / \hbar^{2} .
$$

Upon neglecting the role of the external trap, the width of the ground state, within the gaussian ansatz, is given by $w=(3 / 2) \sqrt{\pi / 2}(1+\sqrt{1+(8 / 3 \pi) s}) \Lambda$, where $\Lambda=\hbar^{2} / m u N$ is the effective "gravitational" radius which fixes the size of the condensate in the absence of $s$-wave interaction $(a=0)$. This expression for $w$ allows for the analytical evaluation of the monopole and quadrupole frequencies (11) and (12) that are plotted in Fig 1 in units of the "gravitational" frequency $m u^{2} N^{2} / \hbar^{3}$. Comparison with the results obtained using the exact ground state shows that the gaussian ansatz provides indeed a good approximation, for both positive and negative values of $s$, provided one is far from the critical value $s_{c} \approx-1$, where the condensate exhibits collapse [1].

The order of magnitude of $\omega_{M}$ and $\omega_{Q}$ is given by the "plasma" frequency $\omega_{p}$, irrespective of the scattering parameter $s$. This can be simply seen by using the virial 
identity (13). Assuming $s>0$ and setting $H_{\text {ho }}=0$ (absence of external trapping), we derive the inequalities $0<2 H_{\text {kin }}<-H_{\text {grav }}$ and, hence, from (11) and (12), we obtain $1<\omega_{M}^{2} / \bar{\omega}^{2}<2$ and $(4 / 5)<\omega_{Q}^{2} / \bar{\omega}^{2}<(14 / 5)$. Here $\bar{\omega}^{2} \equiv-H_{\text {grav }} / N m\left\langle r^{2}\right\rangle$ turns out to be always proportional to the plasma frequency $\omega_{p}^{2}=4 \pi u n / m$. Similarly, assuming $s_{c}<s \leq 0$, we obtain $0<\omega_{M}^{2} / \bar{\omega}^{2} \leq 1$ and $(14 / 5) \leq \omega_{Q}^{2} / \bar{\omega}^{2}<(24 / 5)$. The proximity of the monopole frequency $\omega_{M}$ to the "plasma" frequency $\omega_{p}$ breaks down upon approaching the critical value $s_{c} \approx-1$, where $\omega_{M}^{2} \rightarrow 0\left(\right.$ whereas $\left.\omega_{Q}^{2} \rightarrow(24 / 5) \bar{\omega}^{2}\right)$.

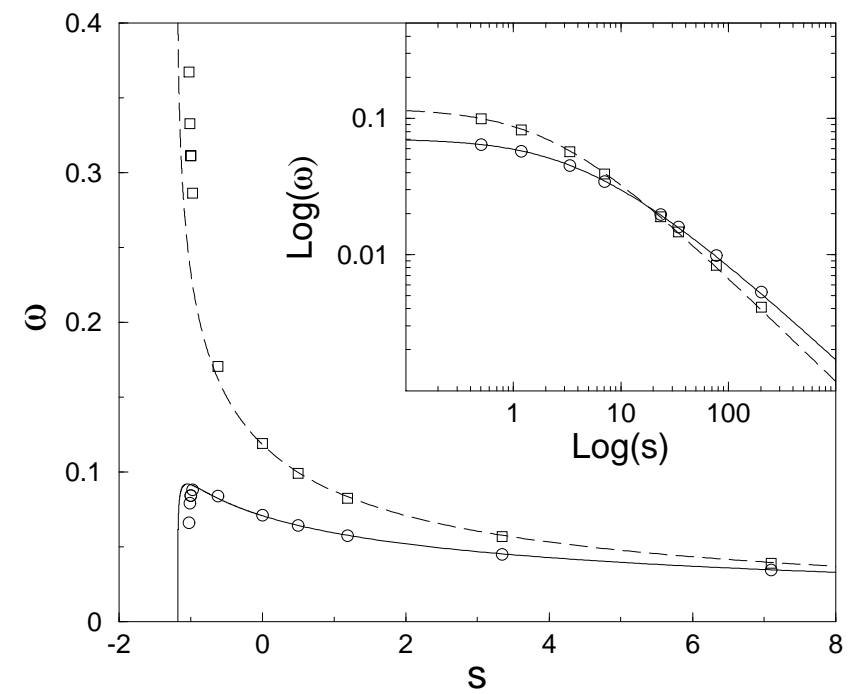

FIG. 1. Monopole and quadrupole frequencies of a self-bound condensate (in $m u^{2} N^{2} / \hbar^{3}$ units) versus the scattering parameter $s=m u N^{2} a / \hbar^{2}$, for both positive and negative values of $a$. Circles: monopole frequencies; squares: quadrupole frequencies. The solid and long-dashed lines correspond to the gaussian approximation. Inset: the corresponding Log-Log plot for $a>0$.

The limits of large and small values of the scattering parameter $s$ (denoted as the TF-G and G self-bound regimes, respectively [1]) will now be discussed separately:

(a) TF-G-regime - In the TF-G-regime (self-bound Thomas-Fermi "gravitational" regime) [1] "gravity" is balanced by the s-wave interaction (large- $s$ limit), while both the kinetic energy $H_{\text {kin }}\left(\propto \hbar^{2}\right)$, and the external trap potential are negligible. In this regime the monopole and quadrupole frequencies in (11) and (12) are given, using the ground state profile (9), by $\omega_{M}=0.6225 \omega_{p}$, $\omega_{Q}=0.3928 \omega_{p}$, where

$$
\omega_{p}=0.5642 m^{\frac{1}{4}} u^{\frac{5}{4}} N^{\frac{1}{2}} \hbar^{-\frac{3}{2}} a^{-\frac{3}{4}}
$$

is the plasma frequency evaluated at the central density. The ratio $\omega_{M} / \omega_{Q}$ turns out to be equal to $\sqrt{5 / 2}$. Remarkably, this is the same result holding for a harmonically trapped BEC in the Thomas-Fermi limit in the absence of the $1 / r$ interaction [9]. In the TF-G regime, the chemical potential $\mu=\left(H_{\mathrm{kin}}+H_{\mathrm{ho}}+2 H_{\mathrm{grav}}+2 H_{\mathrm{s}}\right) / N$ has the value $-\mu=m c_{s}^{2}>>\hbar \omega_{p}$.

It is interesting to note that in the Thomas-Fermi limit the sum-rule expression (11) for the monopole frequency reduces to $\omega_{M}^{2}=2\left|H_{\text {grav }}\right| / N m\left\langle r^{2}\right\rangle$ and coincides with the value derived in politropic stars using classical hydrodynamics [7.

In the TF-G regime the static linear response for the monopole operator $F=\sum_{i=1}^{N} r_{i}^{2}$ can be calculated analytically. This allows for an alternative way to estimate the monopole frequency through the ratio $\hbar^{2} \omega^{2}=m_{1} / m_{-1}$, where the inverse-energy weighted moment $m_{-1}$ is directly related to the static response [12]. In the limit of a small harmonic perturbation $\lambda r^{2}$, the induced density fluctuation takes the analytic form

$$
\delta n=\frac{\lambda}{2 \pi u}\left(\frac{\sin \left(\pi r / R_{0}\right)}{r / \pi R_{0}}-3\right) \theta\left(R_{0}-r\right),
$$

and the inverse-energy weighted moment becomes $m_{-1}=$ $-\delta\left\langle r^{2}\right\rangle / 2 \lambda=\left(6 \pi^{3}-(2 / 5) \pi^{5}\right) m^{-\frac{5}{2}} u^{-\frac{7}{2}} \hbar^{5} a^{\frac{5}{2}}$. In terms of the plasma frequency (15), we finally find $\omega_{M}=$ $0.6181 \omega_{p}$, a value slightly smaller than the one derived from the ratio $m_{3} / m_{1}$.

(b) G-regime — We turn now to the asymptotic Gregime (self-bound "gravitational" regime) [1], in which the s-wave interaction and the external trap potential are negligible, and "gravity" is balanced by the kinetic energy $H_{\text {kin. }}$. In this case the monopole and quadrupole frequencies (11), (12) are given by $\omega_{M}=0.3022 \omega_{p}, \omega_{Q}=$ $0.5057 \omega_{p}$, respectively, where

$$
\omega_{p}=0.2351 m u^{2} N^{2} / \hbar^{3}
$$

is the plasma frequency (2) evaluated at the central density in the same regime. The ratio between these frequencies is now $\omega_{M} / \omega_{Q}=\sqrt{5 / 14}<1$. The chemical potential $\mu=\left(H_{\text {kin }}+H_{\text {ho }}+2 H_{\text {grav }}+2 H_{\mathrm{s}}\right) / N$ in this regime has the value $\mu=-0.6922 \hbar \omega_{p}$. This shows that both the monopole and quadrupole oscillation frequencies are smaller than $-\mu / \hbar$. Consequently, these oscillations are stable against the ejection of atoms from the condensate.

Also in the G-regime the evaluation of the static monopole response can be used to estimate the monopole frequency through the ratio $\hbar^{2} \omega^{2}=m_{1} / m_{-1}$. The calculation yields $\omega_{M}=0.2928 \omega_{p}$. Similarly to the the TF-G regime the monopole frequencies evaluated through the ratios $m_{3} / m_{1}$ and $m_{1} / m_{-1}$ are remarkably close, confirming the validity of the method.

Collisionless hydrodynamics - In the Thomas-Fermi (TF-G) regime, exact results for the oscillation frequencies are obtainable by solving explicitly the equations of collisionless hydrodynamics (5), (6). These results allow us to improve the sum-rule estimate, which in general provides only an upper bound. The equations of motion (5) and (6) can be simplified in this regime, since the quantum pressure term, proportional to $\hbar^{2}$, can be safely ignored. After linearization they yield the equation of motion for the density fluctuations $\delta n$ 


$$
\frac{\partial^{2}}{\partial t^{2}} \delta n=\nabla\left[c_{s}^{2} \nabla(\delta n+\delta \Phi / g)\right],
$$

where the "local" sound velocity $c_{s}(r)=\sqrt{g n(r) / m}$ is calculated at the equilibrium density (9). The time dependence of $\delta n$ is chosen in the form of $\exp (-i \omega t)$, and (18) is transformed, with the help of (4), into

$$
\tilde{\omega}^{2} \tilde{\nabla}^{2} \xi+\tilde{\nabla}\left[\frac{\sin (2 \pi \tilde{r})}{2 \pi \tilde{r}} \tilde{\nabla}\left(\frac{\tilde{\nabla}^{2} \xi}{4 \pi^{2}}+\xi\right)\right]=0,
$$

where we have introduced the "gravitational" fluctuation variable $\xi=\delta \Phi / g$ and used the dimensionless coordinate $\tilde{\mathbf{r}}=\mathbf{r} / \lambda_{J}$ and frequency $\tilde{\omega}=\omega / \omega_{p}$, with $\omega_{p}$ given by (15).

To solve this equation, we set $\xi=\xi_{l}(\tilde{r}) Y_{l m}(\vartheta, \varphi)$, where $Y_{l m}$ are the usual spherical harmonics. The boundary conditions can be derived from the tailoring of the inner and outer solutions on the condensate surface, i.e. at $\tilde{r}=\pi$ [14. The conservation of the total number $N$ of atoms implies that the radial derivative of the potential perturbation vanishes at $\tilde{r} \leq \pi$ for the monopole mode. For the modes with $l=1,2,3, \ldots$ the function $\xi_{l}$ instead decreases as $r^{-(l+1)}$. Hence, the boundary conditions are $\left.\left(d \xi_{0} / d \tilde{r}\right)\right|_{\tilde{r}=\pi}=0$ for $l=0$ and $\left.\left(d \xi_{l} / d \tilde{r}\right)\right|_{\tilde{r}=\pi}=-(l+$ 1) $\xi_{l} / \pi$ for $l>0$ [14].

The numerical solutions of (19) for the monopole and quadrupole frequencies yield $\omega_{M}=0.6168 \omega_{p}, \omega_{Q}=$ $0.392 \omega_{p}$ with $\omega_{p}$ as in (15). Hence, the sum-rule estimates given above, for these frequencies turn out to be very close (within 1\%) to the exact numerical values. Furthermore, they satisfy the inequalities predicted by the general formalism of sum rules [12].

Conclusions - In this paper we have mainly dealt with the oscillations of a "gravitationally" self-bound bosonic cloud, in the framework of the (generalized) Gross-Pitaevskii approach. The Jeans wavelength (8), which is the shortest wavelength to ensure stability, has been shown to be the diameter of the self-bound cloud in the Thomas-Fermi limit [Eq. (90)]. We have derived here simple, analytical expressions for the frequencies of the lowest monopole and quadrupole modes, which are the most relevant collective excitations of such a system, through a sum-rule approach. We have also numerically calculated the eigenfrequencies of the linearized generalized Gross-Pitaevskii equation in the Thomas-Fermi limit of a self-bound BEC and demonstrated that the results are very close to the sum-rule values.

The studied excitations substantially differ from those of harmonically trapped gases interacting via zero-range forces [9, 13]. For harmonically-trapped bosons, all the low-energy excitations (monopole, dipole, quadrupole etc.) are fixed by the oscillator frequency $\omega_{0}$ of the confining trap. By contrast, in the self-bound regimes, only the frequency of the lowest dipole mode, associated with the center of mass oscillations, is fixed by the external trap frequency $\omega_{0}$, whereas all the other low-energy excitations are dominated by the effective "plasma" frequency $\omega_{p}$ (2).
Since the repulsive short-range (s-wave) force can drastically change the size of the self-bound atomic cloud [1], the explicit values of $\omega_{p}$ and the corresponding monopole and quadrupole frequencies are determined by the central density and, consequently, by the regime considered [Eqs. (17) and (15)]. The dependence of the low-frequency collective modes on the plasma frequency resembles the oscillations of compact stars, such as white dwarfs and neutron stars, where the frequency scale $\omega_{p}^{2} \sim 4 \pi G \rho$ provides the universal relation between the oscillation timescale and the mass density $\rho[7]$.

This work was supported by the German-Israeli Foundation (GIF), the Russian Foundation for Basic Research (project 99-02-17076) and the State Program "Universities of Russia" (project 015.01.01.04).

[1] D. O'Dell, S. Giovanazzi, G. Kurizki and V. Akulin, Phys. Rev. Lett. 84, 5687 (2000); see commentary by J. Anglin, Nature 406, 29 (2000).

[2] M.H. Anderson et al., Science 269, 198 (1995); C.C. Bradley et al., Phys. Rev. Lett. 75, 1687 (1995); K.B. Davis et al., Phys. Rev. Lett., 75, 3969 (1995).

[3] For a recent review of the atomic BEC theory see, e.g., F. Dalfovo, S. Giorgini, L.P. Pitaevskii, and S. Stringari, Rev. Mod. Phys. 71, 463 (1999).

[4] S. Giovanazzi, D. O'Dell, and G. Kurizki, quantph/0010045), Phys. Rev. A, Rap. Commun. (in press).

[5] R. Ruffini and S. Bonazzola, Phys. Rev. 187, 1767 (1969); M. Bianchi and D. Grasso, and R. Ruffini, Astron. Astrophys. 231, 301 (1990); G. Ingrosso and D. Grasso and R. Ruffini, Astron. Astrophys. 248, 481 (1991); P. Jetzer, Phys. Rep. 220, 163 (1992).

[6] L.L. Foldy, Phys. Rev. 124, 649 (1961).

[7] S.L. Shapiro and S.A. Teukolsky, "Black Holes, White Dwarfs, and Neutron Stars", (Wiley, New York 1983).

[8] L.P. Pitaevskii, Sov. Phys. JETP, 13, 451 (1961); E.P. Gross, Nuovo Cimento 20, 454 (1961); J. Math. Phys. 4, 195 (1963).

[9] S. Stringari, Phys. Rev. Lett. 77, 2360 (1996).

[10] N. N. Bogoliubov, J. Phys. (U.S.S.R.) 11, 23 (1947).

[11] Frank H. Shu, "The Physics of Astrophysics" (University Science Books, 1992), Vol. 2, Chapter 8.

[12] O. Bohigas, A. M. Lane, and J. Martorell, Phys. Rep. 51, 267 (1979); E. Lipparini and S. Stringari, Phys. Rep. 175, 103 (1989).

[13] V. M. PerezGarcia, H. Michinel, J. I. Cirac, et al., Phys. Rev. Lett. 77, 5320 (1996).

[14] I. E. Mazets, (cond-mat/0007209). 